\title{
Spacing and orientation requirements of GcvA-binding sites 3 and 2 and the Lrp-binding region for gcvT:: lacz expression in Escherichia coli
}

\author{
Lorraine T. Stauffer and George V. Stauffer \\ Author for correspondence: George V. Stauffer. Tel: + 1319335 7791. Fax: + 13193359006. \\ e-mail : george-stauffer@uiowa.edu
}

Department of

Microbiology, University of lowa, lowa City, IA 52242, USA

\begin{abstract}
Both GcvA and Lrp are required for normal regulation of the gcv operon. Moving the GcvA-binding sites 3 and 2 and the Lrp-binding region either closer to, or further away from, the gcv promoter by approximately one helical turn of DNA resulted in a less than twofold decrease in glycine-mediated activation or inosine-mediated repression of a gcvT:: lacz fusion. Moving these sites approximately two helical turns of DNA away from the gcv promoter resulted in a further loss of both activation and repression; moving these sites approximately three helical turns of DNA from the gcv promoter resulted in an essentially complete loss of both glycine-mediated activation and inosinemediated repression. However, when these sites were moved by approximately 1.5 and 2.5 helical turns of DNA away from the gcv promoter, there was a complete loss of both glycine-mediated activation and inosinemediated repression of the gcvT::lacz fusion. The flexibility in the absolute distance of the GcvA- and Lrp-binding sites relative to the gcv promoter, but strict orientation dependence of these sites is consistent with a possible protein-protein interaction of either GcvA, Lrp, or both of these proteins with RNA polymerase. Because of the location of these target sites relative to the gcv promoter, it is also likely that DNA looping is required for this mechanism of regulation.
\end{abstract}

Keywords: glycine cleavage, $g c v T:: l a c Z$, GcvA protein, Lrp protein, regulation

\section{INTRODUCTION}

In Escherichia coli, the conversion of serine to glycine and 5,10-methylenetetrahydrofolate is mediated by the enzyme serine hydroxymethyltransferase $(g l y A$ gene product) and is the major source of one-carbon (C1) units (Mudd \& Cantoni, 1964). The oxidative cleavage of glycine by the glycine-cleavage (GCV) enzyme system provides a second pathway for the production of $\mathrm{C} 1$ units, which are used in the biosynthesis of purines, methionine, thymine and other cellular components (Mudd \& Cantoni, 1964). E. coli mutants deficient in the GCV enzyme system excrete glycine (Plamann et al., 1983); mutants that overproduce the GCV enzyme complex show a reduced growth rate, most likely due to

Abbreviations: GM, glucose minimal; RNAP, RNA polymerase. glycine starvation (Ghrist \& Stauffer, 1995). These observations suggest that the physiological role for the GCV enzyme complex is to balance the requirements for glycine and $\mathrm{C} 1$ units used in numerous biosynthetic reactions.

Numerous proteins have been shown to be involved in the regulation of $g c v$ expression. PurR, a negative regulator of many genes involved in nucleotide metabolism (Kilstrup et al., 1989; Rolfes \& Zalkin, 1988), mediates a twofold repression of the $g c v$ operon in response to purine supplementation (Stauffer \& Stauffer, 1994; Stauffer et al., 1994; Wilson et al., 1993a). Purified PurR protein was shown to bind to a site in the $\mathrm{gcv}$ control region overlapping the transcription start site (Fig. 1a) (Wilson et al., 1993a). Lrp, a global regulatory protein of numerous genes relating to amino acid metabolism (Calvo \& Matthews, 1994), is required for normal induction of the $g c v$ operon (Lin et al., 1992; 
(a)

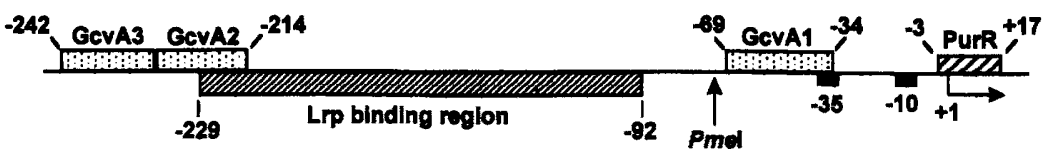

(b)

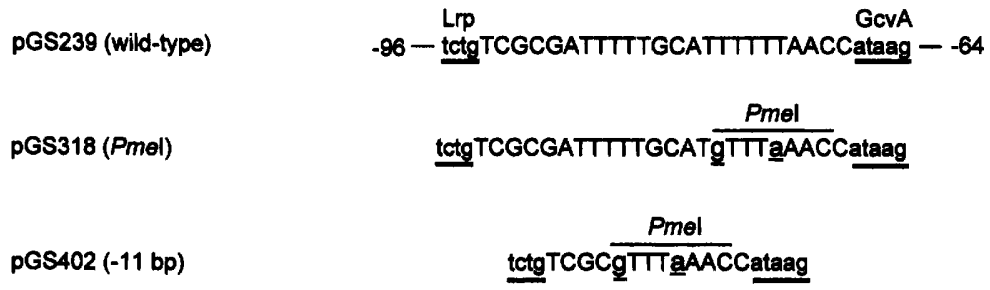

pGS387 (+11 bp) $\quad$ tctgTCGCGATTTTGCATgTTT ggtctagcaccaAACCataag

pGS321 (+14 bp) tctgTCGCGATTTTTGCATgTTTgctctagctagagcaAACCataag

pGS322 (+21 bp) tctgTCGCGATTITGCATgTT cogtctagagcaagctagcaccaAACCataag

pGS323 (+25 bp) tctgTCGCGATTTTTGCATgTTTCgtctagctagagcaagctagcaccaAACCataag

Xbal Tth111 Nhal

pGS391 (+31 bP) tctgTCGCGATTITGCATgTTTcgtctagagogacttgtccaagctagcaccaAACCataag

Fig. 1. (a) The locations of the GrvA upstream (site 3 and site 2) and downstream (site 1) binding sites (Wilson et al., 1995), the Lrp-binding region (Stauffer \& Stauffer, 1994) and the PurR binding site (Wilson et al., 1993a) relative to one another and to the gcv promoter $-35,-10$ and +1 sequences (Stauffer et al., 1993). The position of the Pmel site engineered in this study to facilitate the construction of the insertion and deletion mutations is indicated. (b) Nucleotide sequence of the gcv control region from base -96 to -64 relative to the transcription initiation site. The last four bases of the Lrp-protected region (Stauffer \& Stauffer, 1994) and the first five bases of the GcvA-binding site 1 (Wilson et al., 1995) are underlined. The $-77 \mathrm{G}$ and $-73 \mathrm{~A}$ base changes that generate a unique Pmel site in pGS318 are in lower case and underlined. pGS402 carries an 11 bp deletion from base -78 to -88 . Bases inserted at the Pmel site to generate the $+11,+14,+21,+25$ and +31 insertions are indicated in lower case.
Stauffer \& Stauffer, 1994). Lrp binds to multiple sites upstream of the $g c v$ promoter (Fig. 1a) (Stauffer \& Stauffer, 1994). The GcvA protein activates expression of the $g c v$ operon in glycine-supplemented medium and is also necessary for PurR-independent repression of the operon when cells are grown in the presence of inosine but without glycine (Wilson et al., 1993a, b). GcvA binds to three sites in the $g c v$ control region (Fig. 1a) (Wilson et al., 1995). All three sites are required for normal GcvA-mediated repression of the operon, but only the upstream sites appear necessary for GcvAmediated activation of the operon (Wilson et al., 1995). The GcvR protein negatively regulates the $g c v$ operon (Ghrist \& Stauffer, 1995); its ability to repress is dependent on a functional GcvA protein. Whether GcvR interacts directly with GcvA, with DNA, or perhaps has an enzymic role (for example, synthesizing the corepressor), is unknown.

As described above, the $g c v$ regulatory region is complex, with multiple cis-acting regulatory sites and globaland specific-acting regulatory proteins. We have extended the genetic analysis of this control region by uncoupling the upstream GcvA-binding sites 3 and 2 and the Lrp-binding region from the downstream GcvAbinding site 1 and the gcv promoter element (Fig. 1a). Although the position of the various cis-acting sites shows flexibility in controlling gcv expression, the correct orientation of these sites relative to the $g c v$ promoter is essential. Possible models for the regulatory sites and their binding proteins in expression of the $g c v$ operon are discussed.

\section{METHODS}

Bacterial strains. E. coli $\mathrm{K}-12$ strains used in this study are listed in Table 1.

Table 1. E. coli strains used in this study

\begin{tabular}{|c|c|c|}
\hline Strain* & $\begin{array}{l}\text { Relevant } \\
\text { genotype } †\end{array}$ & Other markers \\
\hline GS162 & Wild-type & $\begin{array}{c}\text { thi phe A905 } \\
\text { AlacU169 } \\
\text { araD129 } \\
\text { rpsL150 }\end{array}$ \\
\hline GS852 & purR6:: Tn10 & as GS162 \\
\hline GS997 & $\operatorname{lrp}: \operatorname{Tn} 10$ & as GS162 \\
\hline GS998 & $g C v A$ & as GS162 \\
\hline GS1053 & $g c \nu R:: \operatorname{Tn} 10$ & as GS162 \\
\hline
\end{tabular}

* GS162 is a derivative of MC4100 (Casadaban, 1976). All other strains were constructed in this laboratory using GS162 as the parent strain.

† The purR6:: Tn10 allele is from strain $\$ \phi 5068$ (Kilstrup et al., 1989). The Irp:: Tn10 allele is from strain CV1008 (Platko et al., 1990). The gcvA (Wilson et al., 1993b) and gcvR::Tn10 (Ghrist \& Stauffer, 1995) mutations were isolated in this laboratory. 
Media. The complex medium used was Luria broth (Miller, 1992). The minimal medium used was the minimal salts of Vogel \& Bonner (1956) supplemented with $0.4 \%$ glucose (GM). GM medium was supplemented with phenylalanine and thiamin since all strains used carry the thi and phe 9905 mutations. Supplements were added at the following concentrations $\left(\mu \mathrm{g} \mathrm{ml}^{-1}\right)$ : glycine, 300 ; inosine, 50; phenylalanine, 50 ; thiamin, 1.

DNA manipulation. The procedures used for restriction enzyme digestion, polymerase chain reactions, etc., were as described by Sambrook et al. (1989). DNA sequencing was by the method of Sanger et al. (1977) using the Sequenase version 2.0 kit (USB).

Mutant construction. pGS239, which carries an in-frame $g c v T$ : : lacZ gene fusion on a 769 bp EcoRI-BamHI fragment (Stauffer et al., 1993), was used as the starting template. Initially, bases -73 and -77 relative to the $g c v$ transcription initiation site were changed to an $A$ and a $G$, respectively, using the PCR megaprimer mutagenesis method (Sarkar \& Sommer, 1990), generating a unique $P m e I$ restriction site. The new plasmid carrying the unique $P m e I$ site was designated pGS318 (Fig. 1b). pGS402 was constructed using the megaprimer mutagenesis method and carries an $11 \mathrm{bp}$ deletion from base -78 to -88 relative to the +1 transcription initiation site (Fig. 1b). pGS322 and pGS391 were constructed by ligating either a $21 \mathrm{bp}$ fragment (containing unique $\mathrm{XbaI}$ and NheI sites), or a $31 \mathrm{bp}$ fragment (containing unique $\mathrm{XbaI}$, Tth111I and NheI sites) respectively, into the PmeI site of pGS318 (Fig. 1b). pGS387 was constructed by digesting pGS391 with $\mathrm{NheI}$ and $\mathrm{XbaI}$, isolating the large fragment from a low-melting-point agarose gel and recircularizing. This resulted in the removal of $20 \mathrm{bp}$ from the original $31 \mathrm{bp}$ insertion (Fig. 1b). pGS321 was constructed by ligating a $10 \mathrm{bp}$ $\mathrm{XbaI}$ linker into the unique $\mathrm{PmeI}$ site of pGS318, digesting with $\mathrm{Xbal}$, filling in the ends with the large fragment of DNA polymerase I and ligating the ends. This resulted in a $14 \mathrm{bp}$ insertion (Fig. 1b). pGS323 was constructed by digesting pGS322 with $\mathrm{XbaI}$, filling in the ends with the large fragment of DNA polymerase I and recircularizing, resulting in a $25 \mathrm{bp}$ insertion at the PmeI site (Fig. 1b). The insertions and deletions were verified by DNA sequence analysis.

Phage construction. An approximately $7 \mathrm{~kb}$ EcoRI-MfeI DNA fragment carrying each $g c v T$ :: lacZ fusion along with the lacY and lacA genes was isolated from each plasmid and ligated into the EcoRI site of $\lambda \mathrm{gt} 2$ (Panasenko et al., 1977) as described previously (Urbanowski \& Stauffer, 1986). The phages generated were single-plaque purified and designated $\lambda g c v T:: \operatorname{lacZ}(\operatorname{Pme} \mathrm{I}), \lambda g c \nu T:: \operatorname{lac} Z(\mathrm{PmeI})-11$, $\lambda g c v T:: \operatorname{lac} Z(P m e \mathrm{I})+11, \quad \lambda g c v T:: \operatorname{lacZ}(\mathrm{PmeI})+14, \quad \lambda g c \nu T::$ $\operatorname{lacZ}(\mathrm{PmeI})+21, \lambda g c v T:: \operatorname{lac} Z(P m e \mathrm{I})+25$ and $\lambda g c v T:: l a c Z$ $(P m e I)+31$. The numbers after $(P m e I)$ indicate the number of deleted or inserted bases at the PmeI site.

Construction of $\lambda$ lysogens. Bacterial strains were lysogenized with the various $\lambda g c v T:: l a c Z$ fusion phages as previously described (Urbanowski \& Stauffer, 1986). All $\lambda$ lysogens carry the cI857 mutation resulting in a temperature-sensitive repressor and were grown at $30^{\circ} \mathrm{C}$. After single-colony purification, lysogens were tested for the presence of only a single copy of $\lambda$ by testing their ability to support lytic infection by $\lambda$ cI90c17 (Shimada et al., 1972).

$\boldsymbol{\beta}$-Galactosidase enzyme assays. $\beta$-Galactosidase activity was assayed as described by Miller (1992) using the chloroform/ SDS lysis procedure. All results are means from two separate assays in which the activity of each sample was determined in triplicate.

\section{RESULTS AND DISCUSSION}

\section{Effects of generating a Pmel site on gcvT::lacz expression}

The $g c v$ control region is complex, with multiple cisacting sites, global and $g c \nu$-specific regulatory proteins (Fig. 1a) (Ghrist \& Stauffer, 1995; Stauffer \& Stauffer, 1994; Wilson et al., 1993a, b, 1995). To gain insight into the roles these sites and their respective DNA binding proteins play in $g c v$ expression, we tested the spacing and geometry of part of the $g c v$ control region on expression of a $g c v T:: l a c Z$ gene fusion. To facilitate these studies, we changed two bases to generate a unique site for the restriction enzyme Pmel. This was done upstream of $g c v$ in a region lacking known binding sites for regulatory proteins (Methods and Fig. 1a). To demonstrate that these two single-base changes do not themselves alter regulation of $g c \nu$, a $\lambda g t 2$ phage carrying the $g c v T:: \operatorname{lac} Z(P m e \mathrm{I})$ mutation was used to lysogenize strains GS852 ( $p u r R)$ and GS998 (gcvA). The lysogens were grown in GM medium with appropriate supplements, and $\beta$-galactosidase levels were measured. The purR lysogen showed about a sixfold glycine-mediated activation and about a fivefold inosine-mediated repression of $g c v T$ : : lac $Z$ expression, similar to the levels measured when the purR strain was lysogenized with a igt2 phage carrying the wild-type $g c v T$ : :lacZ fusion (Table 2). The gcvA lysogen showed a lower basal level of expression in GM medium than the purR lysogen, that was not increased in cells grown in the presence of glycine, nor repressed in cells grown in the presence of inosine (except for an expected twofold repression mediated by PurR) (Wilson et al., 1993a; Stauffer \& Stauffer, 1994; Stauffer et al., 1994).

We also determined the effects of the changes that generate the PmeI site in the lrp mutant strain GS997 and the $g c \nu R$ mutant strain GS1053. In the lrp lysogen grown in GM medium, $\beta$-galactosidase levels were about threefold lower than in the $g c v A$ lysogen; the levels were essentially noninducible by glycine and nonrepressible by inosine (except for an approximately $2 \cdot 5$-fold PurRmediated effect; Table 2). In the $g c v R$ lysogen, $\beta$ galactosidase was constitutively expressed at a high level, although PurR-mediated repression was still observed in cells grown in the presence of inosine (Table 2 ). These results are similar to results reported previously for a wild-type gcvT:: lacZ gene fusion (Stauffer \& Stauffer, 1994; Ghrist \& Stauffer, 1995) and suggest that the changes producing the PmeI site do not significantly alter regulation of the $g c v T$ : : lacZ fusion.

\section{Effects of spacing of GcvA-binding sites 3 and 2 and the Lrp-binding region on gcvT:: lacz expression}

To determine whether the location of the upstream GcvA-binding sites 3 and 2 and the Lrp-binding region relative to the $g c v$ promoter are essential for normal regulation of the gcvT::lacZ fusion, we constructed a series of deletion and insertion mutations of essentially helical turns of DNA at the unique PmeI site in pGS318 (Methods and Fig. 1b). The mutations were cloned into 
Table 2. Effects of changes that create a Pmel site on gcvT:: lacZ expression

Lysogens were grown in GM medium plus the indicated supplements.

\begin{tabular}{|c|c|c|c|c|}
\hline \multirow[t]{2}{*}{ Lysogen } & \multirow[t]{2}{*}{ Relevant genotype } & \multicolumn{3}{|c|}{$\begin{array}{c}\beta \text {-Galactosidase activity } \\
\text { (Miller units) }^{*}\end{array}$} \\
\hline & & None & Glycine & Inosine \\
\hline $852 \lambda g c v T:: l a c Z$ & purR6:: Tn10 & 115 & 672 & 25 \\
\hline 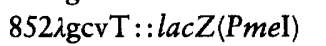 & purR6:: $\operatorname{Tn} 10$ & 92 & 548 & 19 \\
\hline 998גgcvT: :lacZ(PmeI) & $g c \nu A$ & 57 & 60 & 28 \\
\hline 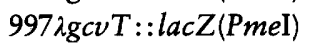 & $\operatorname{lrp}:: \operatorname{Tn} 10$ & 20 & 25 & 8 \\
\hline $1053 \lambda g c v T:: \operatorname{lac} Z\left(P_{m e} \mathrm{I}\right)$ & gcvR:: $\operatorname{Tn} 10$ & 799 & 898 & 550 \\
\hline
\end{tabular}

* All standard deviations were within $10 \%$ of the mean.

Table 3. Effects of deletion and insertion mutations at the Pmel site on gcvT:: lacZ expression

Lysogens were grown in GM medium with the indicated supplements.

\begin{tabular}{|c|c|c|c|}
\hline \multirow[t]{2}{*}{ Lysogen } & \multicolumn{3}{|c|}{$\begin{array}{c}\beta \text {-Galactosidase activity } \\
(\text { Miller units })^{*}\end{array}$} \\
\hline & None & Glycine & Inosine \\
\hline $852 \lambda g c v T:: \operatorname{lac} Z(P m e I)$ & 109 & 652 & 20 \\
\hline $852 \lambda g c v T:: \operatorname{lac} Z(P m e I)-11$ & 68 & 384 & 24 \\
\hline $852 \lambda g c v T:: \operatorname{lacZ}(\mathrm{PmeI})+11$ & 139 & 523 & 49 \\
\hline $852 \lambda g c \nu T:: \operatorname{lac} Z(P m e \mathrm{I})+14$ & 25 & 38 & 20 \\
\hline $852 \lambda g c \nu T:: \operatorname{lac} Z(P m e \mathrm{I})+21$ & 142 & 278 & 93 \\
\hline $852 \lambda g c \nu T:: \operatorname{lac} Z(P m e \mathrm{I})+25$ & 29 & 42 & $20+$ \\
\hline $852 \lambda g c \nu T:: \operatorname{lac} Z(P m e I)+31$ & 136 & 210 & 104 \\
\hline
\end{tabular}

* Standard deviations were within $10 \%$ of the mean except where indicated $(t)$ which was within $18 \%$ of the mean.

$\lambda \mathrm{gt} 2$, which was used to lysogenize the purR strain GS852. The lysogens were grown in GM medium plus appropriate supplements and the $\beta$-galactosidase levels were measured. The -11 lysogen showed slightly reduced levels of expression in GM medium compared

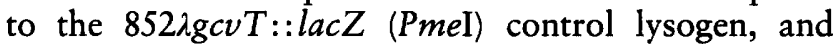
although the levels were induced by glycine, the induced levels were about twofold lower than in the control lysogen (Table 3). The $\beta$-galactosidase levels in the -11 lysogen grown in GM medium containing inosine were not significantly different from the control lysogen. The $11 \mathrm{bp}$ region deleted in this mutant is not protected from DNase I digestion by any known regulatory protein for the gcv operon (Stauffer \& Stauffer, 1994; Wilson et al., 1995). These results suggest that this region does not serve as a cis-acting site for another regulatory protein. The twofold decrease in glycine-mediated activation observed in the -11 lysogen is probably due to the altered spacing of the GcvA-binding sites 3 and 2 and the Lrp-binding region relative to the $g c v$ promoter.
The +11 lysogen showed slightly elevated levels of $\beta$ galactosidase in GM medium compared to the control lysogen, and although glycine induced expression, the induced levels were consistently lower than in the control lysogen (Table 3). In addition, the ability of the GcvA protein to repress expression of the $g c v T:: l a c Z$ fusion in response to inosine was significantly reduced in the +11 lysogen.

The +21 and +31 lysogens showed even more severe effects on gcv $T$ : : lacZ expression (Table 3). In both lysogens, basal levels of expression in GM medium were elevated compared to the control lysogen. In addition, both GcvA-mediated activation in response to glycine and GcvA-mediated repression in response to inosine were reduced significantly, although the effects were greater in the +31 lysogen than the +21 lysogen. These results show that GcvA and Lrp work best from their native positions and work less well when their target sites are moved upstream or downstream relative to the RNA polymerase (RNAP) binding site. Insertions of 11 and 21 bp between the E. coli ilvIH promoter and Lrpbinding region also reduced transcription activation only slightly, whereas Lrp failed to activate when the binding sites were moved far upstream from the promoter (Sacco et al., 1993).

\section{Effects of orientation of GcvA-binding sites 3 and 2 and the Lrp-binding region on gcvT::lacz expression}

We also tested the effects of orientation of the upstream GcvA-binding sites 3 and 2 and the Lrp-binding region on $g c v T:$ : lac $Z$ expression by inserting approximately half-integral turn lengths of DNA at the unique PmeI site in pGS318 (Methods and Fig. 1b). The 14 and $25 \mathrm{bp}$ insertions were cloned into $\lambda \mathrm{gt} 2$ and used to lysogenize the purR strain GS852. The lysogens were grown in GM medium containing appropriate supplements, and $\beta$ galactosidase levels were measured. Both lysogens showed essentially noninducible levels of $\beta$ galactosidase (Table 3). The $\beta$-galactosidase levels, with the exception of those measured in the presence of inosine, were essentially the same as those observed 
Table 4. Effects of Irp, gcvA and gcvR mutations on expression of the $\lambda g c v T::$ lacZ $(P m e l)+21$ insertion

Lysogens were grown in GM medium with the indicated supplements.

\begin{tabular}{|c|c|c|c|c|}
\hline \multirow[t]{2}{*}{ Lysogen } & \multirow[t]{2}{*}{ Relevant genotype } & \multicolumn{3}{|c|}{$\begin{array}{c}\beta \text {-Galactosidase activity } \\
\left(\text { Miller units) }{ }^{*}\right.\end{array}$} \\
\hline & & None & Glycine & Inosine \\
\hline $852 \lambda \mathrm{gcv} T:: \operatorname{lac} Z(P m e \mathrm{I})+21$ & purR6:: Tn10 & 140 & 280 & 93 \\
\hline $998 \lambda g c v T:: \operatorname{lacZ}(P m e \mathrm{I})+21$ & $g c \nu A$ & 100 & 102 & 82 \\
\hline $997 \lambda g c v T:: \operatorname{lacZ}(P m e \mathrm{I})+21$ & $\operatorname{lrp}:: \operatorname{Tn} 10$ & 38 & 46 & 20 \\
\hline $1053 \lambda g c v T:: \operatorname{lacZ}($ PmeI $)+21$ & $g c \nu R:: \operatorname{Tn} 10$ & 246 & 337 & 261 \\
\hline
\end{tabular}

* All standard deviations were within $11 \%$ of the mean.

when the fusion with the wild-type spacing was placed into a lrp background (see Table 2). The higher $\beta$ galactosidase levels observed in medium containing inosine for the +14 and +25 lysogens compared to the lrp control lysogen are due to the presence of PurR in the lrp lysogen (Stauffer et al., 1994; Stauffer \& Stauffer, 1994; Wilson et al., 1993a). These results suggest that both activation and repression of the $g c v T::$ lacZ fusion are face-of-the-helix dependent and require a precise stereospecific orientation of either GcvA, Lrp or both, relative to the $g c v$ promoter. A stereospecific alignment between the $i l v I H$ and the gltBDF promoters and their upstream Lrp-binding sites is also needed for activation (Sacco et al., 1993; Wiese et al., 1997).

\section{Effects of Irp, gcvA and gcvR mutations on expression of the $\lambda g c v T$ ::lacz(Pmel) +21 fusion}

We also tested the effects of $\operatorname{lrp}, g c v A$ and $g c v R$ mutations on expression of the +21 fusion. We chose the +21 bp insertion phage as it still shows about a twofold glycine-mediated induction of the $g c v T:: l a c Z$ fusion (Table 3). The phage was used to lysogenize the $g c v A$ strain GS998, the $l r p$ strain GS997 and the $g c v R$ strain GS1053. The new lysogens, along with the original $852 \lambda$ gcv $T::$ lacZ $(P m e \mathrm{I})+21$ lysogen, were grown in GM medium with appropriate supplements, and $\beta$ galactosidase levels were determined. The $g c \nu A+21$ lysogen was noninducible by glycine and nonrepressible by purines, except for a small effect due to the PurR protein (Table 4). These results indicate that the approximately twofold glycine-dependent induction in the purR +21 lysogen is dependent on GcvA. In the lrp +21 lysogen grown in GM medium, the $\beta$-galactosidase levels were reduced about threefold compared to the $g c v A+21$ lysogen (Table 4). The levels were essentially noninducible by glycine and nonrepressible by inosine, except for the twofold PurR-mediated repression. The $g c v R+21$ lysogen showed constitutive expression of $\beta$ galactosidase, although the PurR-mediated repression was still observed in the presence of inosine. The GcvR protein is a negative regulator of $g c v T:: l a c Z$ expression and its ability to repress is dependent on a functional
GcvA protein (Ghrist \& Stauffer, 1995). It was therefore possible that the altered regulation observed when the GcvA-binding sites 3 and 2 and the Lrp-binding region were moved closer to or further from the $g c v$ promoter was due to an alteration in GcvR-mediated regulation. This seems unlikely, however, since both activation and repression are reduced in the insertion mutants. In addition, in the $g c v R+21$ lysogen, the constitutive levels of expression are higher than in the control $852 \lambda g c v T:: \operatorname{lac} Z(P m e \mathrm{I})+21$ lysogen, but the levels are still about threefold lower than in the 1053igcvT::lacZ(PmeI) control lysogen (compare Tables 2 and 4). Thus, the reduced levels of expression observed from the deletion or insertion of helical turns of DNA is most likely due to a failure to activate the $g c v T::$ lacZ fusion rather than to an increased ability of GcvR to negatively regulate the $g c \nu T:: l a c Z$ fusion.

\section{Working models}

The location of GcvA-binding sites 3 and 2 and the Lrpbinding region relative to the $g c v$ promoter (Fig. 1a), as well as the strict requirement that GcvA and Lrp proteins bind on the correct surface of the DNA relative to RNAP for both activation and repression, make it likely that DNA looping is part of the mechanism of $g c v$ operon regulation. Lrp is known to bend DNA (Wang \& Calvo, 1993) and a previous DNase I footprint analysis of Lrp binding to the $g c v$ control region DNA indicated that Lrp might cause bending of the DNA (Stauffer \& Stauffer, 1994). In addition, it was shown previously that GcvA-binding sites 3 and 2 are required for both activation and repression of the $g c v$ operon, whereas GcvA-binding site 1 is required only for repression (Wilson et al., 1995). Thus, an Lrp-induced DNA bend might allow GcvA bound at sites 3 and 2 to interact with RNAP to activate the $g c v$ promoter, or possibly to interact with GcvA bound at site 1 to repress the $g c v$ promoter. Since GcvR requires a functional GcvA protein for its negative effect (Ghrist \& Stauffer, 1995), GcvR might function in this model to influence which type of protein-protein interaction occurs as a result of the DNA loop, or the degree of the DNA bend. However, 
it has not been shown at this time whether GcvR has a direct or an indirect role in $g c v$ operon control. It is also possible that Lrp directly interacts with RNAP, or is required for cooperative binding with GcvA protein. We have purified the Lrp protein and are currently purifying the GcvA and GcvR proteins. These proteins will be used in in vitro systems to test their effects on cooperativity in binding to their respective target sites and for their ability to activate or repress $g c v$ in a run-off transcription assay.

\section{ACKNOWLEDGEMENTS}

This investigation was supported by Public Health Service grant GM26878 from the National Institute of General Medical Sciences.

\section{REFERENCES}

Calvo, J. M. \& Matthews, R. G. (1994). The leucine-responsive regulatory protein, a global regulator of metabolism in Escherichia coli. Microbiol Rev 58, 466-490.

Casadaban, M. J. (1976). Transposition and fusion of the lac genes to selected promoters in Escherichia coli using bacteriophage lambda and Mu. J Mol Biol 104, 541-555.

Ghrist, A. C. \& Stauffer, G. V. (1995). Characterization of the Escherichia coli gcvR gene encoding a negative regulator of $g c v$ expression. J Bacteriol 177, 4980-4984.

Kilstrup, M., Meng, L. M., Neuhard, J. \& Nygaard, P. (1989). Genetic evidence for a repressor of synthesis of cytosine deaminase and purine biosynthesis enzymes in Escherichia coli. $J$ Bacteriol 171, 2124-2127.

Lin, R., D'Ari, R. \& Newman, E. B. (1992). $\lambda$ placMu insertions in genes of the leucine reguion: extension of the regulon to genes not regulated by leucine. J Bacteriol 174, 1948-1955.

Miller, J. H. (1992). A Short Course in Bacterial Genetics. Cold Spring Harbor, NY: Cold Spring Harbor Laboratory.

Mudd, S. H. \& Cantoni, G. L. (1964). Biological transmethylation, methyl-group neogenesis and other 'one-carbon' metabolic reactions dependent upon tetrahydrofolic acid. In Comprehensive Biochemistry, pp. 1-47. Edited by M. Florkin \& E. H. Stotz. Amsterdam: Elsevier.

Panasenko, S. M., Cameron, J. R., Davis, R. W. \& Lehman, I. R. (1977). Five hundredfold overproduction of DNA ligase after induction of a hybrid lambda lysogen constructed in vitro. Science 196, 188-189.

Plamann, M. D., Rapp, W. D. \& Stauffer, G. V. (1983). Escherichia coli $\mathrm{K} 12$ mutants defective in the glycine cleavage enzyme system. Mol Gen Genet 192, 15-20.

Platko, J. V., Willins, D. A. \& Calvo, J. M. (1990). The ilvIH operon of Escherichia coli is positively regulated. J Bacteriol 172, 4563-4570.
Rolfes, R. J. \& Zalkin, H. (1988). Escherichia coli gene purR encoding a repressor protein for purine nucleotide synthesis. J Biol Chem 263, 19653-19661.

Sacco, M., Ricca, E., Marasco, R., Paradiso, R. \& Felice, M. D. (1993). A stereospecific alignment between the promoter and the cis-acting sequence is required for Lrp-dependent activation of ilvIH transcription in Escherichia coli. FEMS Microbiol Lett 107, 331-336.

Sambrook, J., Fritsch, E. F. \& Maniatis, T. (1989). Molecular Cloning: a Laboratory Manual, 2nd edn. Cold Spring Harbor, NY: Cold Spring Harbor Laboratory.

Sanger, F., Nicklen, S. \& Coulson, A. R. (1977). DNA sequencing with chain-terminating inhibitors. Proc Natl Acad Sci USA 74, 5463-5467.

Sarkar, G. \& Sommer, S. S. (1990). The 'megaprimer' method of site-directed mutagenesis. Biotechniques 8, 404-407.

Shimada, K., Weisberg, R. A. \& Gottesman, M. E. (1972). Prophage lambda at unusual chromosomal locations. I. Location of the secondary attachment sites and the properties of the lysogens. $J$ Mol Biol 63, 483-503.

Stauffer, L. T. \& Stauffer, G. V. (1994). Characterization of the gcv control region from Escherichia coli. J Bacteriol 176, 6159-6164.

Stauffer, L. T., Ghrist, A. \& Stauffer, G. V. (1993). The Escherichia coli gcvT gene encoding the $\mathrm{T}$-protein of the glycine cleavage enzyme system. DNA Seq-J DNA Seq Mapping 3, 339-346.

Stauffer, L. T., Fogarty, S. J. \& Stauffer, G. V. (1994). Characterization of the Escherichia coli gcv operon. Gene 142, 17-22.

Urbanowski, M. L. \& Stauffer, G. V. (1986). Autoregulation by tandem promoters of the Salmonella typhimurium LT2 metJ gene. J Bacteriol 165, 740-745.

Vogel, H. J. \& Bonner, D. M. (1956). Acetylornithinase of Escherichia coli: partial purification and some properties. J Biol Chem 218, 97-106.

Wang, Q. \& Calvo, J. M. (1993). Lrp, a global regulatory protein of Escherichia coli, binds co-operatively to multiple sites and activates transcription of ilvIH. J Mol Biol 229, 306-318.

Wiese, D. E., Ernsting, B. R., Blumenthal, R. M. \& Matthews, R. G. (1997). A nucleoprotein activation complex between the leucineresponsive regulatory protein and DNA upstream of the gltBDF operon in Escherichia coli. J Mol Biol 270, 152-168.

Wilson, R. L., Stauffer, L. T. \& Stauffer, G. V. (1993a). Roles of the GcvA and PurR proteins in negative regulation of the Escherichia coli glycine cleavage enzyme system. J Bacteriol 175, 5129-5134.

Wilson, R. L., Steiert, P. S. \& Stauffer, G. V. (1993b). Positive regulation of the Escherichia coli glycine cleavage enzyme system. J Bacteriol 175, 902-904.

Wilson, R. L., Urbanowski, M. L. \& Stauffer, G. V. (1995). DNA binding sites of the LysR-type regulator GcvA in the $g c v$ and $g c v A$ control regions of Escherichia coli. J Bacteriol 177, 4940-4946.

Received 22 October 1997; revised 5 January 1998; accepted 12 January 1998. 